\title{
les coulées de boue du Freney, près de Modane, en 1977 et 1978
}

\author{
par \\ C. Azimi \\ P. Desvarreux \\ P. Plotto \\ Association pour le Développement des Recherches sur les Glissements de Terrains
}

\section{Introduction}

Aux printemps de 1977 et 1978 , des coulées de boue et de blocs sont survenues dans le talus naturel dominant la RN 6, un peu avant le village du Freney en Maurienne. Ces phénomènes ont intéressé un versant boisé, haut d'une centaine de mètres, incliné à $37-40^{\circ}$, et sur une longueur totale de $350 \mathrm{~m}$ environ. Sur ce versant aucun phénomène analogue n'avait été mentionné auparavant. L'étude entreprise pour le compte de la Subdivision de Modane (D.D.E.) avait pour but de préciser les conditions dans lesquelles s'étaient produites les coulées ainsi que les travaux à envisager.

\section{Coulées de 1977 et 1978}

La RN 6 étant située à $1020 \mathrm{~m}$ environ, deux arrachements se sont produits au printemps 1977, I'un vers $1175 \mathrm{~m}$ d'altitude, avec sortie d'eau visible, l'autre vers 1080-1100 m sans sortie d'eau. Ils se sont traduits par des mouvements locaux sur une épaisseur de quelques mètres, une largeur de 20 à $30 \mathrm{~m}$ et une longueur identique, mais sans formation de coulée.

Du 19 au 22 Mai 1977 se sont produits trois arrachements successifs avec coulées de boue et blocs, ayant démarré vers $1040-1060 \mathrm{~m}$ d'altitude et qui ont intéressé une largeur de $80 \mathrm{~m}$ au total, et une épaisseur de $2 \mathrm{~m}$ environ de terrain. A chaque arrachement correspondait une venue d'eau qui est devenue bien visible après le départ des coulées et qui est restée établie par la suite.

Simultanément, une grande fissure est apparue, partant de l'extrémité supérieure de ces arrachements, côté Modane, et remontant vers $1090 \mathrm{~m}$ pour rejoindre horizontalement un des arrachements survenus avant le 19 Mai 1977.

(A la suite d'une première étude, des travaux de drainage ont été entrepris par la Subdivision de Modane en 1977 et ont été complétés en 1978.)
En 1978, le 25 Février, une coulée importante survenait côté La Praz, sur 40 à $50 \mathrm{~m}$ de large, et s'étendant de la R.N. 6 jusqu'à $1100 \mathrm{~m}$ d'altitude, avec venues d'eau visibles vers $1050 \mathrm{~m}$ d'altitude.

A la même époque, un glissement s'amorçait côté Modane sur $100 \mathrm{~m}$ de large environ, remontant à $60-70 \mathrm{~m}$ au-dessus de la route. Ce glissement s'accélérait en Avril 1978 en donnant encore des coulées.

Pratiquement tout le versant dominant la RN 6 sur $100 \mathrm{~m}$ de haut et $350 \mathrm{~m}$ de long, a été le siège en 1977 ou 1978 de glissements et coulées intéressant une épaisseur de 2 à $3 \mathrm{~m}$ de terrain, et accompagnés de sorties d'eau localisées réparties sur divers niveaux.

Plusieurs remarques peuvent être faites :

- d'après les Services des Eaux et Forêts et ceux de l'Équipement, rien de tel ne s'était produit à cet endroit depuis longtemps. Cependant, en examinant des photographies aériennes de 1959, on pouvait observer un arrachement ayant emporté le terrain de la même manière, sur 10 à $15 \mathrm{~m}$ de large, ayant démarré à $50 \mathrm{~m}$ environ au-dessus de la RN 6:

- d'après les Services des Eaux et Forêts, les deux ruisseaux de Savoutier et du Sartay, qui encadrent le site étudié donnent lieu de temps à autre à des coulées boueuses:

- d'après l'examen de la pluviométrie journalière à Modane (fig. 1 et 2), on constate que certaines des coulées (19 au 22 Mai 1977) peuvent être corrélées avec de très fortes pluies, mais pas les autres (en particulier celles du 25 Février 1978, Mai et Juillet 1978).

L'étude entreprise a donc été orientée sur l'hydrogéologie du secteur et ses modifications ayant pu déclencher ce phénomène. Elle a compris des observations géologiques et hydrogéologiques, des reconnaissances géophysiques, une opération de multitraçage et des mesures de résistivité de l'eau. 


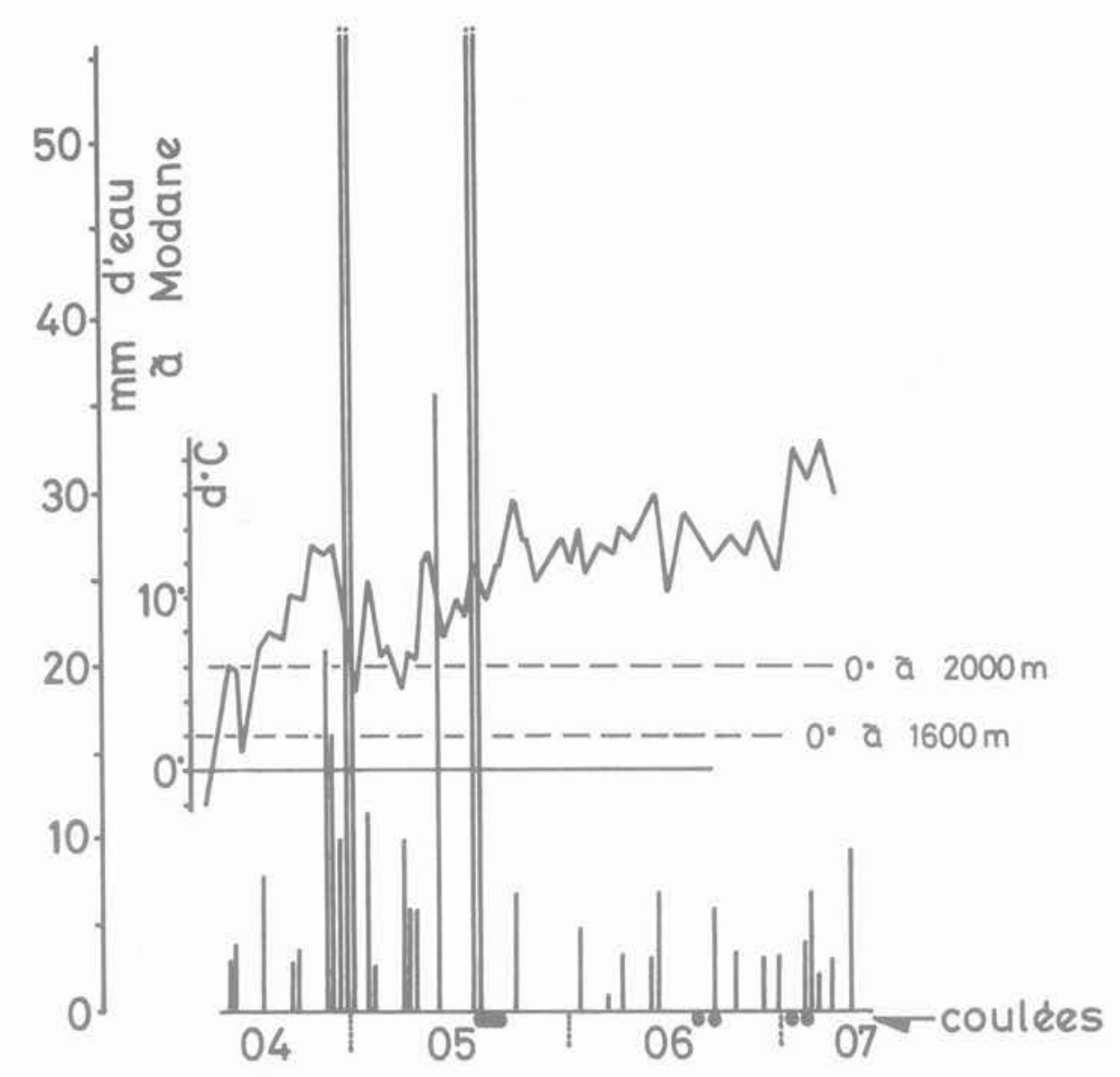

Fig. 1 Pluviométries et températures en 1977

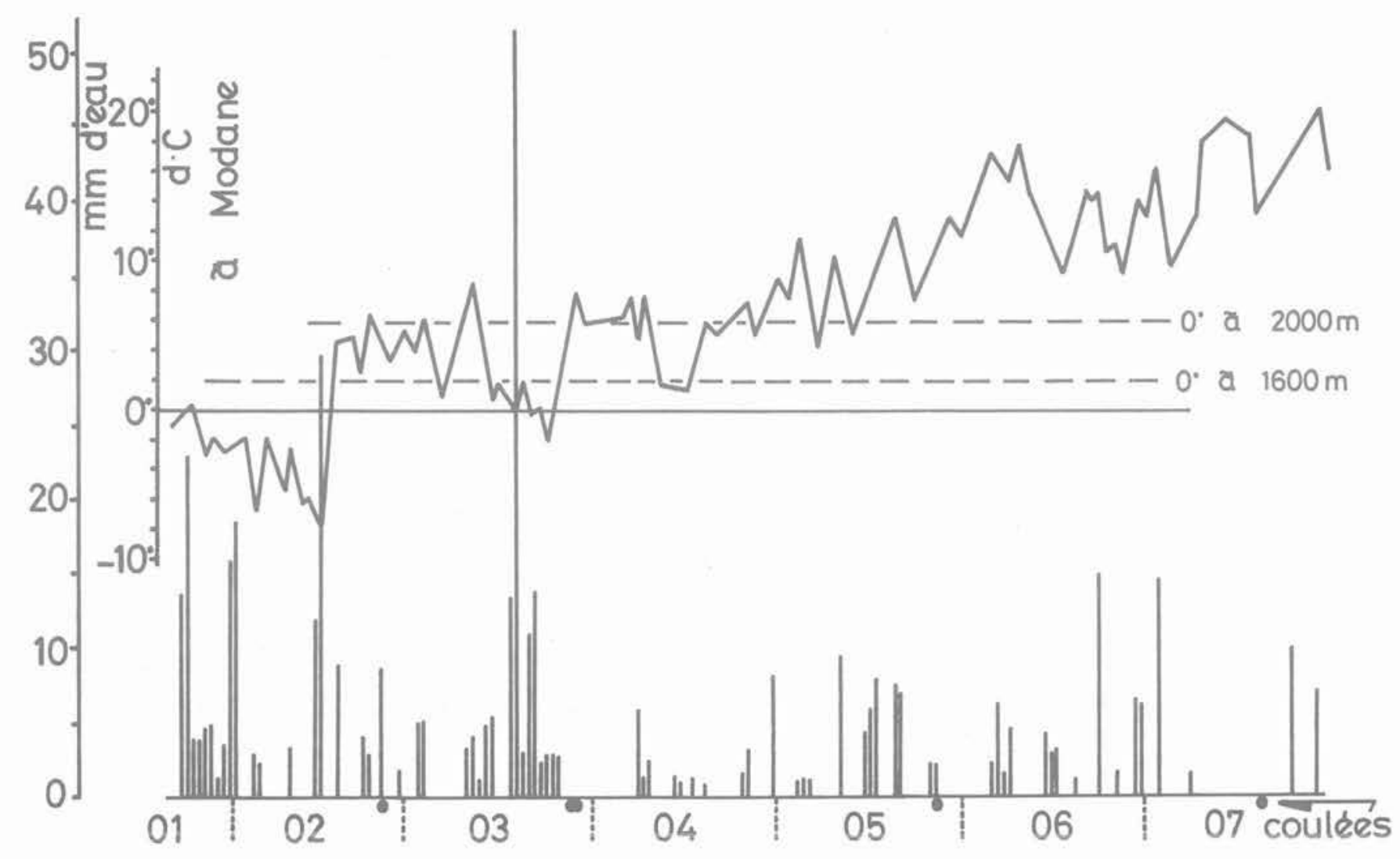

Fig. 2 Pluviométries et températures en 1978 


\section{Principales observations géologiques}

Le versant qui domine la RN 6 a été étudié jusqu'au replat des Dérozes à $1950 \mathrm{~m}$ d'altitude. On peut y distinguer de haut en bas (fig. 3 ) :

- une zone supérieure formée de falaises rocheuses fracturées, de pente moyenne $50^{\circ}$ (de 1900 à $1600 \mathrm{~m}$ d'altitude),

- de 1600 à $1500 \mathrm{~m}$ une zone d'éboulis récents de pente 20 à $30^{\circ}$,

- de 1500 à 1350 m une zone boisée de $35^{\circ}$ de pente,

- de 1350 à $1150 \mathrm{~m}$ une zone en pente plus douce $\left(25^{\circ}\right)$ autrefois couverte de prairies,

- de 1150 à $1000 \mathrm{~m}$ la pente de $37-40^{\circ}$ dominant la RN 6.

Cette succession irrégulière de pentes et l'examen des photographies aériennes montre que tout le versant, jusqu'au replat des Dérozes, correspond à un glissement ancien de grande importance, ayant intéressé une épaisseur de l'ordre de $100 \mathrm{~m}$ de rocher. La largeur totale de ce glissement est de 800 m environ et le secteur qui a donné naissance aux coulées représente son pied $\mathrm{E}$.

Ce secteur est à présent limité par 2 torrents :

- le ruisseau de Savoutier côté W,

- le ruisseau de Sartay côté E.

Le substratum rocheux est formé de grès et de schistes gréseux et micacés du houiller. On l'observe dans l'ancienne niche d'arrachement entre 1600 et $1900 \mathrm{~m}$ d'altitude. Les couches de direction NS ont un pendage de $15^{\circ}$ vers I'W. Elles sont affectées de fauchage sur une épaisseur de 5 à $15 \mathrm{~m}$, ce qui provoque des éboulements alimentant les éboulis vifs qu'on trouve en-dessous.
Dans le reste du versant, n'affleurent que des terrains de couverture, à savoir :

- des éboulis proprement dits formés de blocs de grès jusqu'à plusieurs $\mathrm{m}^{3}$ et de schistes dans une matrice silto-argileuse. Cette matrice peut être plus ou moins argileuse selon la nature de la roche d'origine (schistes anthraciteux ou non);

- des éboulis à base de rocher très fissuré où on peut observer encore des paquets de couches de 10 à $15 \mathrm{~m}$.

\section{Reconnaissances géophysiques}

Les profils sismiques et électriques avaient pour but de rechercher éventuellement un substratum en place, d'une part, et d'autre part de préciser les hétérogénéités des terrains de couverture.

Les trois profils géophysiques ont été exécutés, le $1^{\text {er }}$ à $50 \mathrm{~m}$ au-dessus de la RN 6, après départ des coulées, le $2^{\circ}$ à $80-100 \mathrm{~m}$ au-dessus de la RN 6 au sommet des arrachements, le $3^{\circ}$ à $120 \mathrm{~m}$ environ au-dessus de la RN 6 sur une piste forestière. Ils ont compris des profils sismiques de $110 \mathrm{~m}$ de long réalisés à l'explosif ( 5 tirs) avec un enregistreur à 12 pistes (en collaboration avec I'I. R. I. G. M.) et des sondages électriques réalisés selon le dispositif de Wenner avec $A B_{\max }=72 \mathrm{~m}$, les centres des sondages étant distants de $20 \mathrm{~m}$.

Sur le profil inférieur, la sismique permet de repérer à $10-15 \mathrm{~m}$ de profondeur un substratum caractérisé par $\mathrm{V}_{e}=2160 \mathrm{~m} / \mathrm{s}$, ce qui indique du rocher fissuré, surmonté de 2 couches l'une à $1450 \mathrm{~m} / \mathrm{s}$, l'autre à $1000 \mathrm{~m} / \mathrm{s}$. Les résistivités varient de 400 à $1200 \Omega \cdot \mathrm{m}$ sur les 3 à 5 premiers mètres puis diminuent ( 200 à $400 \Omega \cdot \mathrm{m}$ ). On peut interpréter ces données comme indiquant la possibilité d'existence d'une nappe $\left(\mathrm{V}_{e}=1450 \mathrm{~m} / \mathrm{s}\right)$ dans la partie basse du versant à $4 \mathrm{ou}$ $5 \mathrm{~m}$ de profondeur.

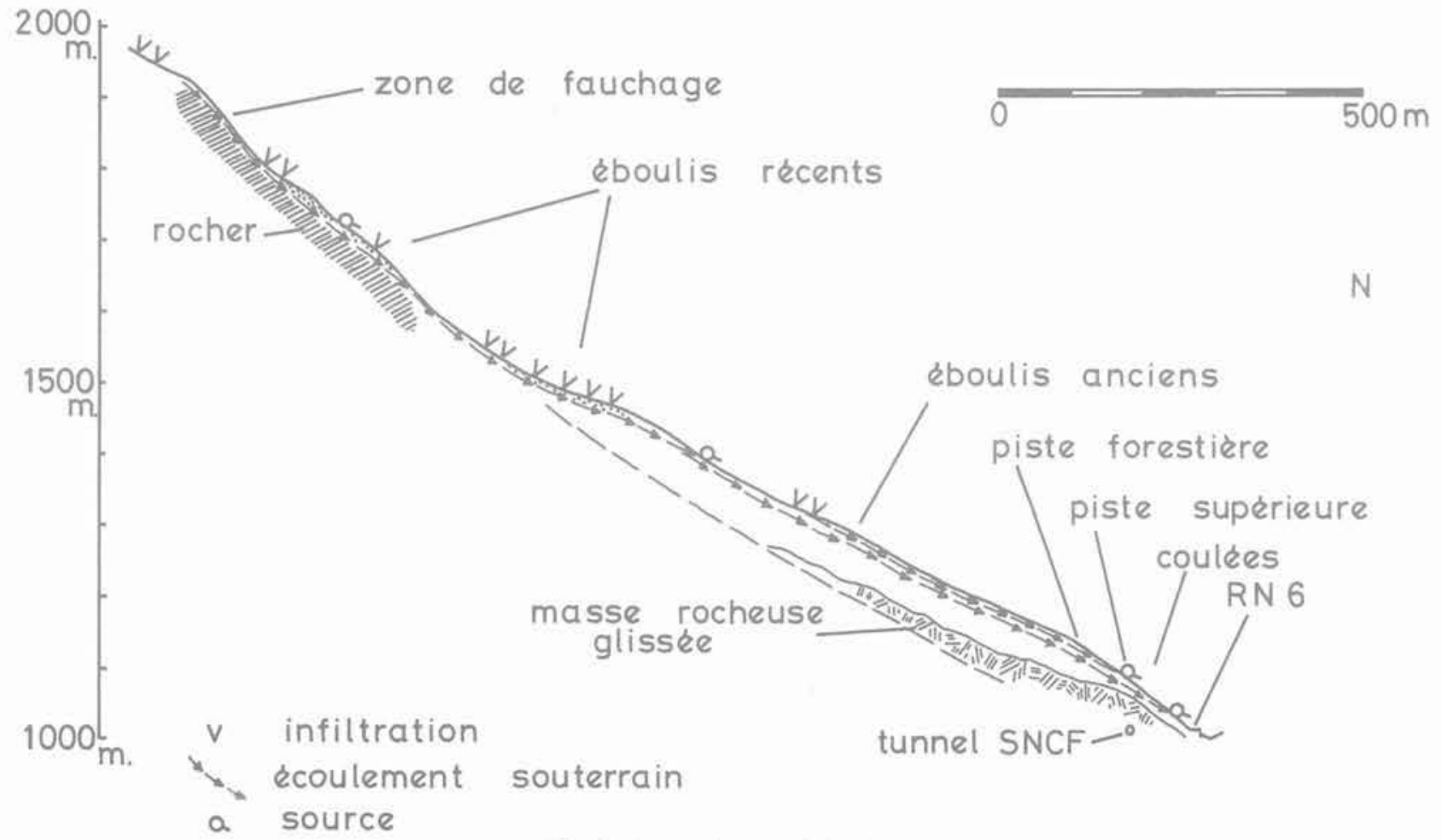

Fig. 3 Coupe hydrogéologique 


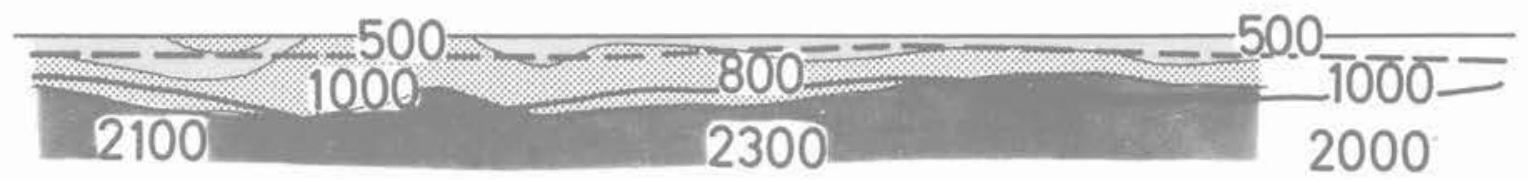

E
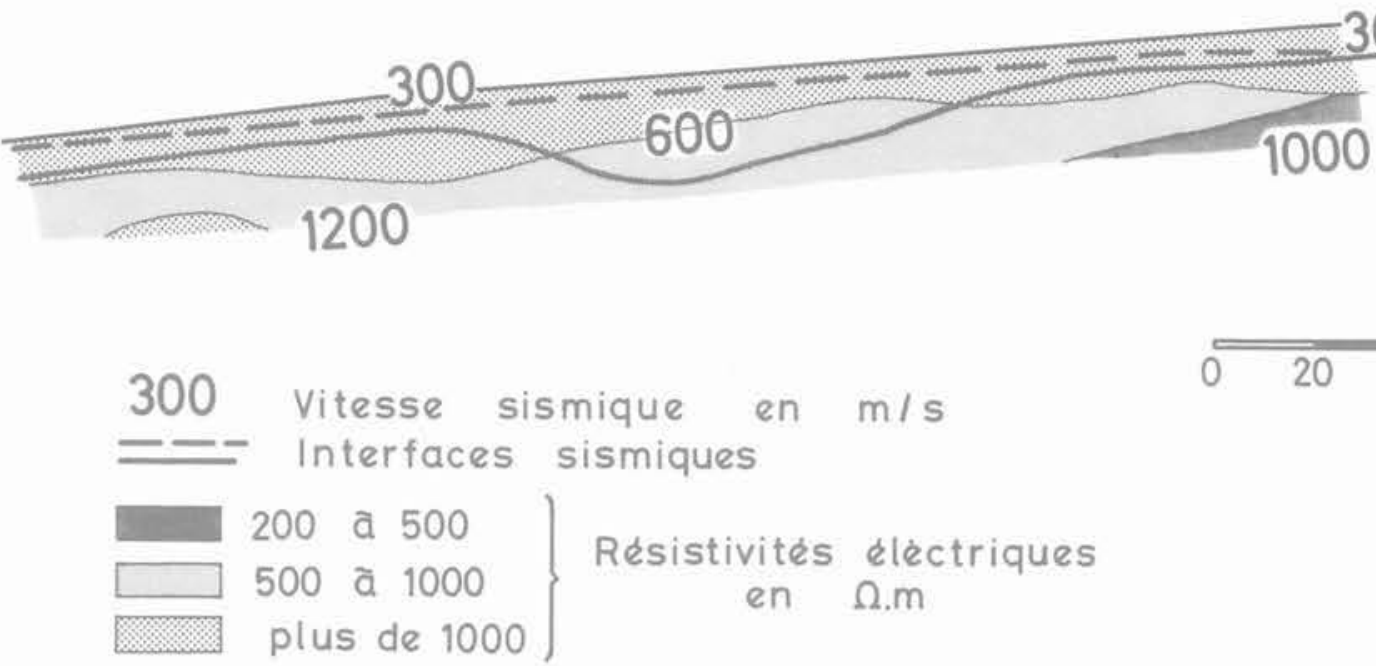

Fig. 5 Profil géophysique sur la piste forestière

Sur le profil intermédiaire, la sismique permet de repérer un substratum à $2000-2300 \mathrm{~m} / \mathrm{s}$ à une profondeur de 7 à $20 \mathrm{~m}$. Au-dessus on trouve une couche à $740-1070 \mathrm{~m} / \mathrm{s}$, puis sur 2 à $5 \mathrm{~m}$ en surface une couche à $420-500 \mathrm{~m} / \mathrm{s}$. Les résistivités correspondant au substratum sismique sont de 180 à $600 \Omega \cdot m$ Au-dessus elles augmentent (1600 à $2400 \Omega \cdot m$ ) indiquant que la partie inférieure des éboulis n'est vraisemblablement pas saturée. Enfin, dans la tranche superficielle de 1 à $5 \mathrm{~m}$, les résistivités sont variables de $400-600 \Omega \cdot m$ à $3000 \Omega \cdot m$, indiquant la possibilité de circulations d'eau localisées dans cette tranche (fig. 4).

Enfin, sur le profil supérieur, la sismique ne repère plus de substratum, lequel doit se situer à plus de 35-40 m de profondeur, ou bien est très brisé, car les vitesses sont de 1070 à $1330 \mathrm{~m} / \mathrm{s}$. Les terrains de couverture sont peu compacts puisqu'on trouve une couche à $500-600 \mathrm{~m} / \mathrm{s}$ jusqu'à $20 \mathrm{~m}$ de profondeur au maximum. Ces éboulis ont des résistivités de 1200 à $2000 \Omega \cdot \mathrm{m}$, ce qui exclut la présence d'une nappe d'eau. La couche sous-jacente a une résistivité de 500 à $800 \Omega \cdot \mathrm{m}$. Elle peut correspondre soit à de l'éboulis avec matrice argileuse, soit au substratum fissuré (fig. 5).

Ces reconnaissances ont donc permis de préciser qu'il n'existe pas de nappe dans le versant, tout au moins pas à moins de 20 ou $30 \mathrm{~m}$ de profondeur environ, sinon on aurait des mouvements d'ensemble de tout le versant, y compris la RN 6. Mais le rocher fissuré est le siège de circulations d'eau comme le prouvent les observations faites sur le tunnel S.N.C.F. Enfin, en partie basse ( 20 à $30 \mathrm{~m}$ dominant la route) il semble exister une nappe à 4 ou $5 \mathrm{~m}$ de profondeur. Elle n'a été repérée que dans la partie Est et peut correspondre aux venues d'eau par le rocher fissuré.
Par conséquent, les venues d'eau observées lors des coulées ne peuvent être associées à une nappe dont le niveau serait remonté. Leur origine a été éclaircie par les diverses observations hydrogéologiques.

\section{Observations hydrogéologiques}

Les observations faites sur le terrain:

\section{1 Écoulements superficiels}

Les principaux sont les deux ruisseaux de Savoutier et de Sartay qui prennent naissance dans les arrachements supérieurs vers 1600 à $1900 \mathrm{~m}$ d'altitude, par suite de la réunification de petits ruisseaux. Plusieurs observations sont importantes :

- le ruisseau de Savoutier, qui au printemps 1978 coulait jusqu'à l'Arc, se perdait en Septembre 1978 dans son propre lit, à $1440 \mathrm{~m}$ d'altitude;

- d'après les photos aériennes de 1970, il semble que le ruisseau de Savoutier alimentait en fait le ruisseau de Sartay par "capture * vers $1500 \mathrm{~m}$ d'altitude;

- fin Juin 1978 , le ruisseau de Sartay, en crue par suite de la fonte des neiges, est sorti de son lit vers $1350 \mathrm{~m}$ d'altitude et une partie des eaux s'est déversée en rive gauche pour atteindre l'extrémité W des arrachements dominant la RN 6;

- on observe enfin plusieurs niveaux de pertes dans le versant. L'une correspond à la zone d'éboulis récents entre 1500 et $1650 \mathrm{~m}$ d'altitude (voir fig. 6). L'autre correspond à la cote 1300-1350 m entre les 2 ruisseaux : plusieurs écoulements, provenant de sources, se perdent à ce niveau. 


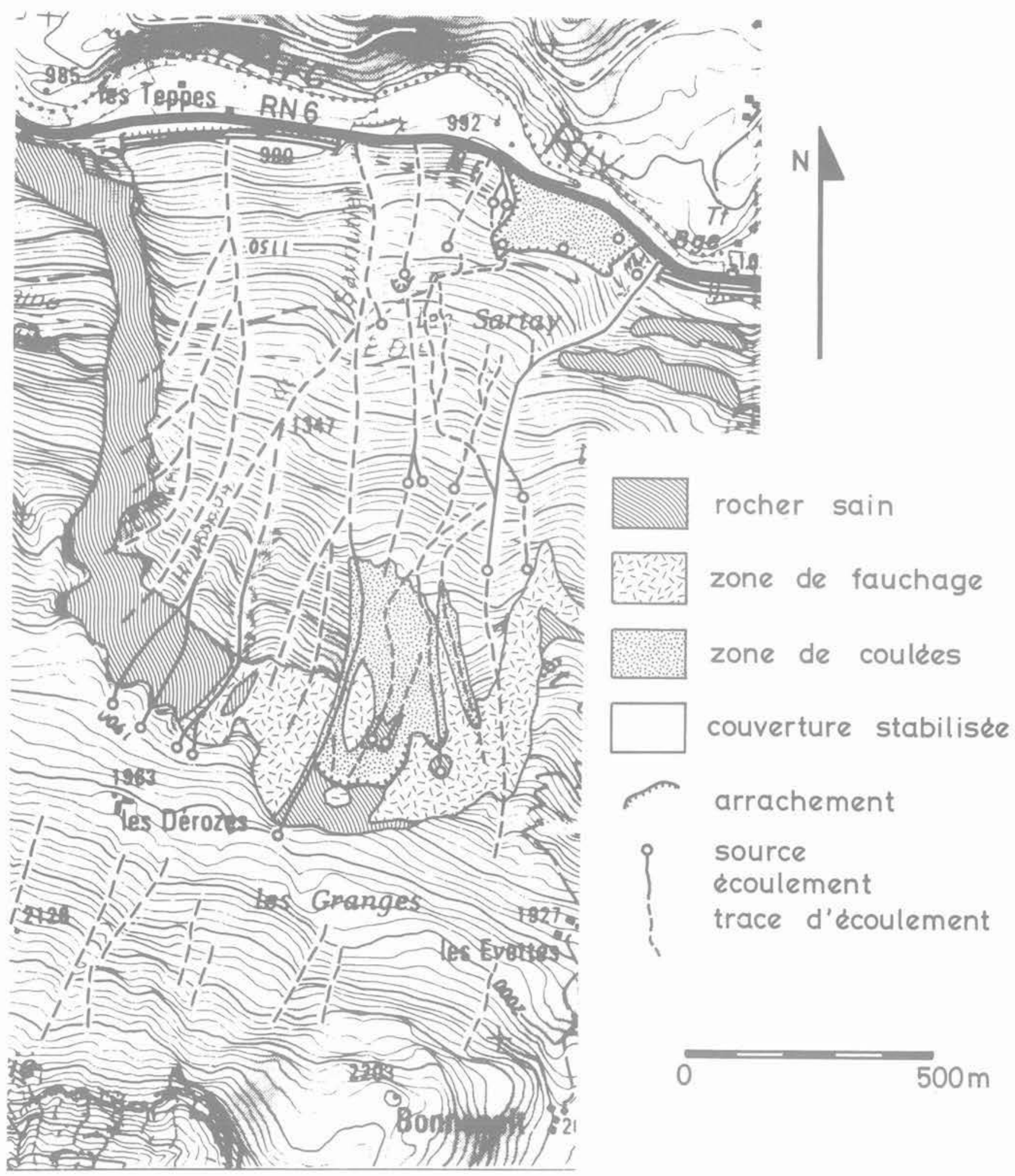

Fig. 6 Carte géologique

\subsection{Sources}

Tous les mouvements survenus en 1977 et 1978 ont fait apparaître des sources, invisibles auparavant à cet endroit du versant. Le premier niveau de ces sources est situé à $30-50 \mathrm{~m}$ au-dessus de la RN 6. D'autres sources existent actuellement à cette cote dans la partie de terrain n'ayant pas bougé, côté La Praz. Le deuxième niveau de sources apparues lors des coulées est situé à $80-100 \mathrm{~m}$ au-dessus de la RN 6.
D'autres sources existent dans le versant, notamment l'une à $1175 \mathrm{~m}$ d'altitude, liée à un glissement superficiel, et d'autres vers $1380 \mathrm{~m}$ d'altitude, entre les 2 ruisseaux. Les écoulements de ces dernières se perdent $50 \mathrm{~m}$ plus bas (voir plus haut).

\section{3 Écoulements d'eau à faible profondeur}

On a de plus des preuves d'existence de circulations d'eau (ou des possibilités de telles circulations) à faible 
profondeur, localisées dans des chenaux plus perméables :

- lors de l'exécution d'une piste pour les drainages, dans la partie supérieure des arrachements, on a observé que certaines arrivées d'eau correspondaient à des passages très perméables formés de blocs remplissant des chenaux profonds de 1 à $2 \mathrm{~m}$, creusés dans l'éboulis, et situés juste sous la surface du sol:

- l'une des pertes décrites au paragraphe précédent, à $1300 \mathrm{~m}$ d'altitude, se situe dans un talweg, qui est sec en aval de la perte, et rempli de végétation. En suivant ce talweg ( $\propto$ talweg central $n$ ), nous avons abouti à la source située à $1175 \mathrm{~m}$ d'altitude, laquelle a donné lieu en 1977 à un glissement localisé. II y a donc très probablement un écoulement souterrain dans ce talweg sur $350 \mathrm{~m}$ de long, et à très faible profondeur.

\section{Opération de multitraçage et mesures annexes}

A ce stade de l'étude nous avions suffisamment d'éléments pour supposer que les sources apparues lors des coulées étaient alimentées par des circulations localisées dans des chenaux perméables, enterrés à plus ou moins grande profondeur. Le problème était de préciser comment avaient pu être alimentés ces chenaux, et à quel endroit. Pour cela, nous avons réalisé, en collaboration avec I'Institut Dolomieu, une opération de traçage par plusieurs colorants utilisés simultanément, et une série de mesures de résistivité de l'eau.

\subsection{Multitraçage}

Le multitraçage a été réalisé du 27 Octobre 1978 au 8 Novembre 1978, ce qui correspond à une période d'étiage où les colorants ne sont pas excessivement dilués. On a injecté 3 colorants (fig. 7 ):

- du bichromate de potassium au point I, correspondant à une infiltration locale à $1350 \mathrm{~m}$, en RG du torrent de Sartay,

- de la fluoresceine au point II, correspondant à l'infiltration repérée dans le talweg central à $1330 \mathrm{~m}$ d'altitude,

- de la rhodamine WT a la perte du ruisseau de Savoutier, à $1440 \mathrm{~m}$ d'altitude.

On a choisi 5 points de prélèvement correspondant à des sources de la cote 1050, ou de la cote 1100, ou enfin à la source située au débouché du *talweg central s.

La rhodamine n'a été retrouvée nulle part. Le bichromate est réapparu au bout de 12 à $14 \mathrm{~h}$ au point 4 correspondant aux sources déjà captées à la cote 1100 (concentration 1 à $2 \cdot 10^{-7}$ pour une injection de $500 \mathrm{~g}$ pour $10 \mathrm{\ell}$ ). La fluoresceine est réapparue au point 1 au bout de 12 à $13 \mathrm{~h}$, et au point 5 au bout de $11 \mathrm{~h}$. Les concentrations mesurées ont été faibles $\left(5 \cdot 10^{-10}\right.$ pour une injection de $600 \mathrm{~g}$ pour $4 \ell$ de solution alcoolisée).

On peut immédiatement tirer plusieurs conclusions :

- il est possible de préciser la correspondance entre les zones d'infiltration et les zones de sortie d'eau (fig.7). En particulier les eaux ressortant vers $1050 \mathrm{~m}$ ne proviennent pas de l'un des 3 points d'infiltration choisis, alors que celles ressortant vers $1100 \mathrm{~m}$ proviennent soit du point $\mathrm{I}$, soit du point II. II y a donc plusieurs écoulements indépendants et superposés;

- les temps de parcours permettent de calculer les vitesses d'écoulement dans le terrain. On arrive à $30 \mathrm{~m} / \mathrm{h}$ ou $0,8 \mathrm{~cm} / \mathrm{s}$. Ceci confirme clairement qu'il s'agit de chenaux très perméables. En effet les éboulis sont des sols de la classe GM, à granulométrie étalée contenant 12 à $20 \%$ de fines $(<0,1 \mathrm{~mm})$. Pour de tels matériaux, l'ordre de grandeur de perméabilité est de $10^{-4}$ à $10^{-7} \mathrm{~cm} / \mathrm{s}$ (Sherard et al, Earth and earth-rock dams, 1967).

\subsection{Mesures de résistivité}

On a mesuré les résistivités de l'eau en divers points du versant. Les résultats sont donnés à la figure 8 , et permettent de confirmer les résultats du traçage. En effet les eaux du site ont des résistivités de 30 à $40 \Omega \cdot \mathrm{m}$ ce qui correspond à une faible minéralisation. Mais l'eau se minéralise au fur et à mesure qu'elle circule dans le terrain, ce qui entraîne une diminution de sa résistivité.

Par exemple, pour le cheminement repéré entre les points II et 5 , sur une longueur de $330 \mathrm{~m}$, la résistivité de l'eau passe de $44,8 \Omega \cdot \mathrm{m}$ à $38,3 \Omega \cdot \mathrm{m}$. On retrouve un gradient analogue entre les points l et 4 (diminution de la résistivité de 39,7 à $31,5 \Omega \cdot m$ pour $400 \mathrm{~m}$ de parcours).

Si on considère donc que dans de tels chenaux le gradient de résistivité ramené à $1 \mathrm{~m}$ de parcours est en moyenne de $0,02 \Omega \cdot \mathrm{m} / \mathrm{m}$, on peut conclure que l'eau du point $2(\rho=35 \Omega \cdot m)$ ne peut provenir d'infiltration au point $\mathrm{I}$, situé environ à $500 \mathrm{~m}$, car la résistivité de l'eau devrait y être de $45 \Omega \cdot m$ au moins. De même, I'eau ressortant à proximité de la RN $6(\rho=21,5 \Omega \cdot \mathrm{m})$ provient à priori de zones d'infiltrations plus lointaines car elle est plus fortement minéralisée.

\section{Synthèse des données. Mécanisme proba- ble des coulées}

A ce nouveau stade de l'étude, on peut déjà tirer un certain nombre de conclusions.

- Les terrains compris entre les ruisseaux de Savoutier et de Sartay sont des terrains anciennement glissés dont la limite supérieure remonte à $1900 \mathrm{~m}$ d'altitude. Ces terrains sont formés, en profondeur, de paquets rocheux (schistes et grès) fracturés, avec circulations d'eau dans les fissures (tout au moins en partie basse du versant). Ces masses rocheuses sont recouvertes de 10 à $50 \mathrm{~m}$ d'éboulis de schistes et grès. Ceux-ci deviennent de plus en plus argileux vers la base du versant (diminution des résistivités) où il semble exister une nappe à 4 ou $5 \mathrm{~m}$ de profondeur. Le reste des éboulis n'est pas saturé et contient un certain nombre de chenaux perméables vraisemblablement indépendants. Ces chenaux débouchent dans le talus naturel dominant la RN 6 sur $100 \mathrm{~m}$ de haut, à des altitudes différentes.

- On a pu mettre en évidence le lien existant entre deux zones d'infiltration visibles à $1300-1350 \mathrm{~m}$ et plusieurs sources situées à 1100-1175 m d'altitude dont certaines ont donné lieu à des coulées en 1977-78. L'origine des sources apparues vers $1050 \mathrm{~m}$ lors des coulées de 1977 n'a pas été prouvée mais il est logique de penser qu'elles sont 


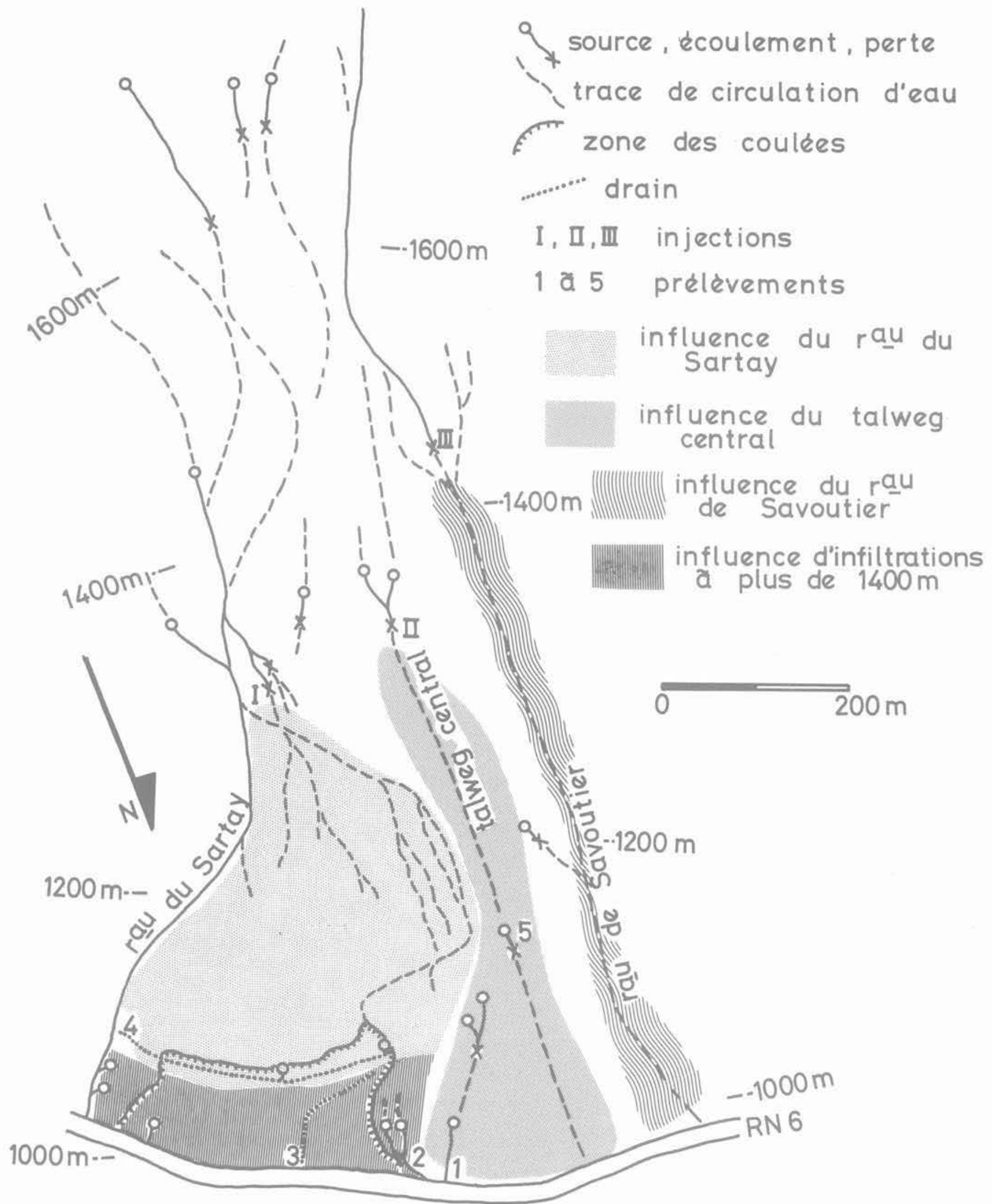




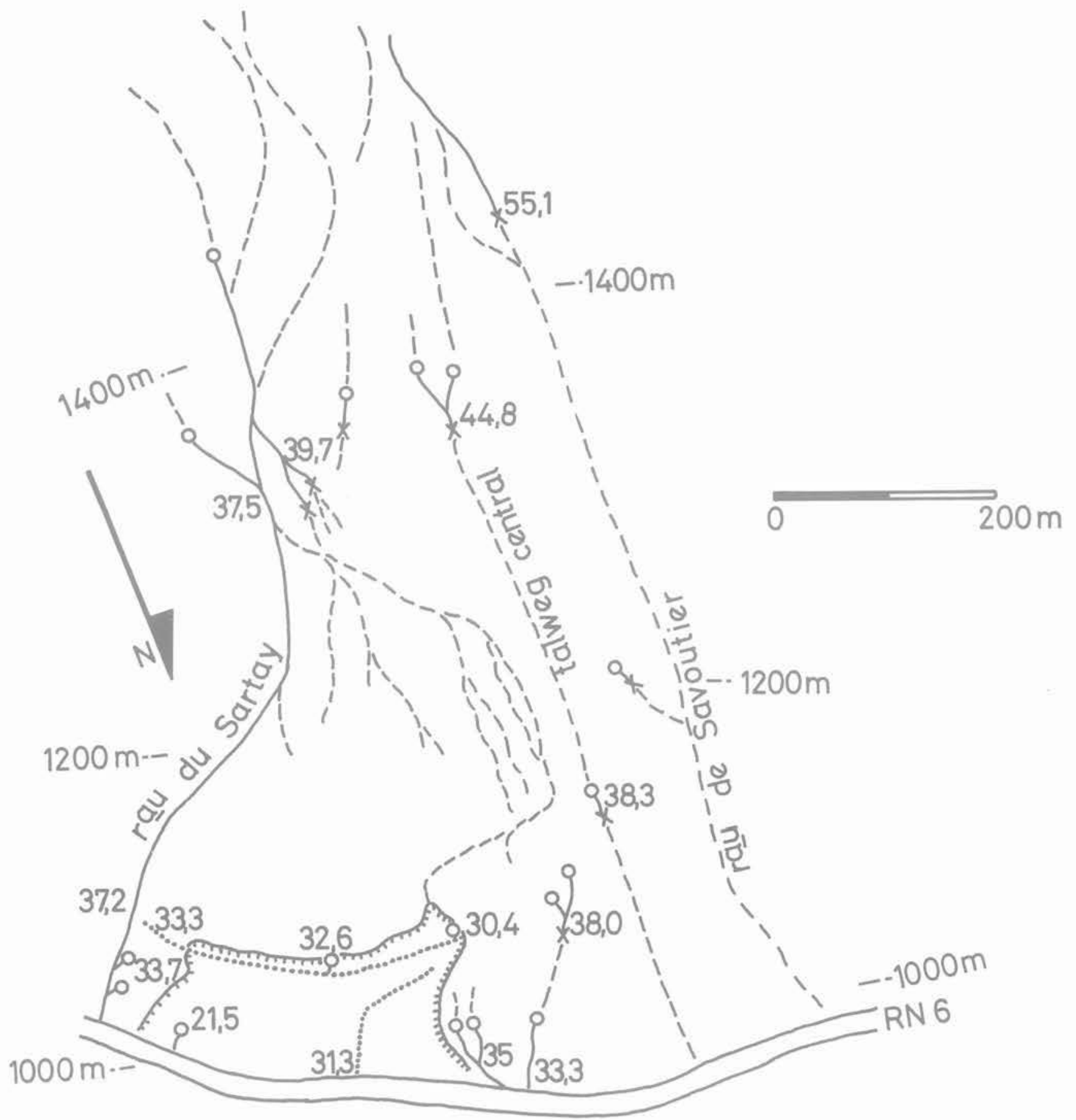

Fig. 8 Résistivités de l'eau en $\Omega m$

liées à des infiltrations dans les éboulis vifs vers 1500-1650 m (voir coupe fig. 3).

Comme avant 1977, dans la zone des coulées, aucun écoulement n'était visible, on peut supposer que les coulées sont dues à une brusque augmentation de débit dans les chenaux aboutissant aux sources, le débit initial étant nul ou très faible. Les sorties normales de ces chenaux étaient recouvertes de 2 à $3 \mathrm{~m}$ de terre végétale et d'éboulis récents. C'est cette épaisseur de terrain qui s'est mise en mouvement sous l'effet de l'augmentation de pression interstitielle.

Comment ces chenaux ont-ils pu se trouver alimentés brutalement? La raison peut être trouvée, à notre avis, dans l'observation de plusieurs faits :

- si les hivers $1976-77$ et 1977-78 ne sont pas exceptionnels sur le plan de la pluviométrie, on peut néanmoins corréler un certain nombre de coulées soit avec de fortes pluies, soit avec des redoux très marqués, en particulier les coulées du 25-02-1978 et 28 et 30-03-1978. On a alors une crue des torrents pouvant entraîner leur débordement ou même leur divagation, d'où des infiltrations aléatoires pouvant aboutir à des chenaux préexistants; 
- en partie haute, l'activité des éboulements dans l'ancienne niche d'arrachement a augmenté depuis quelques années, d'après les Eaux et Forêts. II y a, dans la zone d'éboulis vifs entre 1500 et $1650 \mathrm{~m}$ d'altitude de nombreuses traces de divagations (lits abandonnés), de dépôts de coulées boueuses n'ayant pas été plus bas (ce qui fait qu'au niveau de la RN 6 seules quelques coulées épisodiques ont été observées dans les lits des ruisseaux). Dans ces dépôts de coulées on trouve des troncs d'arbres, qui peuvent former des barrages temporaires s'ils se mettent en travers et permettent ainsi des changements de lit.

On peut alors expliquer de la manière suivante les coulées de 1977 et 1978. Au printemps 1977, plusieurs coulées ont dû se produire en partie haute, entraînant des changements de lit des deux ruisseaux et des infiltrations. L'eau a ainsi envahi des chenaux perméables débouchant vers $1050 \mathrm{~m}$, au-dessus de la RN 6 ou elle a fini par déclencher les grosses coulées des 19 et 22 Mai 1977. La longueur de parcours étant de $900 \mathrm{~m}$, si les vitesses moyennes étaient de $30 \mathrm{~m} / \mathrm{h}$, le temps nécessaire est de $30 \mathrm{~h}$. On peut donc lier ces coulées aux très fortes pluies du 18 et 19 Mai, ayant provoqué une augmentation de débit brutale. Les coulées de Juin et Juillet 1977 peuvent être liées à la fonte des neiges au-dessus de $2000 \mathrm{~m}$ d'altitude.

La coulée du 25 Février 1978 peut être mise en correspondance avec un redoux très marqué à partir du 17 Février, entraînant une fonte des neiges au moins jusqu'à $1600 \mathrm{~m}$ d'altitude. C'est le cas aussi des coulées des 28 et 30 Mars. Mais en 1978 on sait de plus que le ruisseau de Sartay est sorti de son lit vers $1300-1350 \mathrm{~m}$ d'altitude et on sait que les infiltrations à ce niveau aboutissent à diverses sources de la cote $1100 \mathrm{~m}$. Ceci expliquerait que les coulées de 1978 ont démarré, en général, plus haut que celles de 1977 .

On constate ainsi que le phénomène est d'origine naturelle, et qu'il peut être aléatoire car les infiltrations dépendent des débordements. Ces derniers peuvent être liés soit à l'augmentation de débit, soit à des barrages temporaires provoqués, dans les lits des ruisseaux, par des matériaux de petites coulées boueuses.
On peut alors conclure sur le principe des travaux qui seraient les plus efficaces soit pour empêcher l'extension du phénomène, soit pour empêcher qu'il se reproduise.

- Captage de toutes les sources mises en évidence après le départ des coulées, dans le but d'éviter l'érosion régressive.

- Captage des sources actuellement visibles vers 1050-1100 m d'altitude et situées à l'W de la zone des coulées.

- Captage des écoulements superficiels visibles vers $1300 \mathrm{~m}$ d'altitude et situés entre les ruisseaux de Savoutier et de Sartay pour diminuer les infiltrations dans le terrain.

- Travaux de rectification du lit du ruisseau de Sartay entre 1200 et $1360 \mathrm{~m}$ pour éviter de nouveaux débordements à l'avenir.

Un partie de ces travaux a déjà été réalisée par les Services de I'Équipement de Modane, et depuis l'été 1978 la partie basse est stabilisée.

\section{Remerciements}

Nous remercions vivement Monsieur Bailly, Ingénieur TPE à Modane, pour l'aide apportée à la réalisation de l'étude ainsi que pour l'autorisation de publication des résultats obtenus.

Nous remercions également Monsieur Fourneaux, de I'Institut Dolomieu, et Monsieur Simonnet, de I'I. R.I.G. M., pour leur collaboration aux opérations de multitraçage et de prospection sismique.

\section{Référence bibliographique}

Sherard J.L. et al (1963) «Chap. 4. Explorations for foundations and embankment construction materials". Earth and Earth-rock Dams. $3^{\circ}$ ed. John Wiley and Sons Inc. 1967. p. 269. 
